\title{
El emblematismo en la poesía espańola y novohispana del barroco: Luis de Sandoval Zapata
}

Rocío Olivares Zorrilla

Facultad de Filosofía y Letras

Universidad Nacional Autónoma de México

Es con frecuencia aceptado en los medios académicos que de los múltiples vasos comunicantes que unen literatura y artes visuales, la emblemática ofrece un repertorio icónico e ideológico insoslayable en la reconstrucción del sentido de la obra artística de los siglos XVI al XviII, sentido cuya integridad los pasos de la historia han difuminado haciendo invisible lo que antes tenía la función de hacernos ver lo imperceptible. De acuerdo con M. J. Woods, en el caso de la poesía barroca, de carácter fundamentalmente descriptivo - aun dentro de lo que pudiera concebirse como un transcurso narrativo-, la ékphrasis morosa se convierte en densidad metafórica que conduce a la paradoja (42). Más aún, podría decirse que, por vía del emblematismo, la alegoría, con sus alcances metafísicos, es la meta más encarecida del sentido poético, el cual se esparce en las modulaciones metafóricas e incluso epitéticas, cuando no recoge en el seno del discurso poético, en el sistema del poema, todo un conjunto de significados que el emblema en sí mismo ha ya sistematizado y sostenido en la figura.

Los poetas barrocos, fascinados siempre por el mundo natural, concibieron sus líricas persuasiones morales, sus exempla sobre lo temporal y lo eterno inspirados en el Eclesiastés, como un paradójico homenaje a la naturaleza temporal como creación divina. La Epistola a los romanos (Tapié: 45) es otra fuente de la idea del mundo como libro, y los Ejercicios de san Ignacio de Loyola contribuirán a hacer de esta convicción, también agustiniana, uno de los paradigmas del barroco. Alain Tapié, 
en su libro sobre el sentido oculto de las flores, observa que, en el siglo XVII, aun el conocimiento natural estaba compenetrado con la simbolización, como lo estaban la alquimia y la física. En el caso del arte, el poder del símbolo revelaba a los ojos del lector o del espectador la autonomía de la mímesis artística, subrayándola y evidenciándola (34). La acumulación de objetos inanimados de las representaciones manieristas ya explotaba esta semantización deliberada de los elementos más triviales de la vida cotidiana que, dentro de la obra artística, se convertían en un mensaje cifrado. A este respecto hay que mencionar el famoso cuadro flamenco de Rubens y Jan Brueghel titulado "Guirnalda con la Virgen y el Niño", que González de Zárate (282-290) ha decodificado de manera brillante mostrando cómo lo que en apariencia es ornamental se convierte en semiosis autosuficiente. Esta pintura es una muestra clara de la pauta ecléctica del manierismo, irradiada de Italia y de los Países Bajos, que impregnó el arte en todas sus manifestaciones. Una de ellas, el ars topiaria o arte de la jardinería, convertía los jardines españoles en receptáculo de múltiples influencias, siendo la italiana una de las más vigorosas, fusionada con la jardinería árabe anterior al jardín renacentista. De acuerdo con Morales Folguera, $(304,315-316)$ la fuente textual en España para el diseño y construcción de los grandes jardines cortesanos fue el Sueño de Polifilo o Hypnerotomachia, de Francesco Colonna, prolijamente comentado por él en relación con los jardines de El Retiro, en Málaga. Junto a la Arcadia de Sannazaro, la obra de Colonna había inspirado, desde el siglo xv, la jardinería de Florencia, Tívoli y Bomarzo. En España, las vívidas descripciones de las escenas por las que atraviesa Polifilo en su trayectoria a la isla Citérea fueron traducidas no sólo en Málaga, sino en la Casa de Campo de Madrid y en otros jardines que, como el de Pedro Soto de Rojas en Granada, reproducían, estancia a estancia, el ascenso del alma a la cúspide contemplativa de la naturaleza. La reflexión neoestoica ante el espectáculo natural es comentada, a su vez, por Rubén Pardo Lesta al referirse a la obra de Soto de Rojas, a la que aludiré más adelante a propósito del poeta novohispano Luis de Sandoval Zapata. Señala Pardo Lesta (1012-1013) que el ascenso a la cima de un monte para contemplar la creación y aproximarse por esa vía al Creador está en la epístola que Petrarca dirige al rey Filipo de Macedonia. En ella Petrarca relata cómo, al extender la vista desde lo alto, abre al azar las Confesiones de san Agustín, en cuyas páginas lee sobre el olvido de sí mis- 
mo que experimentan al admirar la enormidad de la creación divina en las alturas de las montañas. Hay otros notables precedentes de esta idea en el pensamiento hispánico, anterior o contemporáneo a Petrarca, como El filósofo autodidacta, de Abentofáil, y el Tratado del amor de las criaturas, de Ramón Sibiuda, que ya he comentado en otra parte (Olivares 2000: 96). ${ }^{1}$ Ya en el contexto del barroco, Alain Tapié menciona la obra del jesuita flamenco Leroy d'Alard sobre "la santidad de la vida desprendida de la consideración de las flores”. ${ }^{2}$ La Compañía de Jesús hizo suyo este tipo de reflexión de Leroy d'Alard que ya venía de la obra del abad Suger (Goody: 175), así como de las monjas beguinas y los místicos alemanes del siglo XIV. El papel de los jardines espirituales como verdadero género literario prohijado por la orden jesuita se extendió desde Amberes y Lovaina a Lyon, la Valencia francesa, Boloña, Roma y Nápoles (Tapié: 45). Desde principios del siglo xvi, la metáfora de la Virgen María como jardín - hortus conclusus, fons signatus_- en la interpretación de san Jerónimo del Cantar de los Cantares (Falkenburg: 17) —convirtió al inventario floral de los jardines en un auténtico repertorio emblemático- También Tapié menciona la polisemia de las flores, observando que la diversidad de significados llega a ser contradictoria en algunos casos (43). Por si fuera poco, el distinto desenvolvimiento de la emblematización floral en los países católicos y los protestantes suprimió la posibilidad de un sentido único, y en esa medida la interpretación tendía a ser libre ubicándose en la mirada del contemplador. Una flor podía tener un significado para la tradición cristiana, basado en el principio del amor; otro para la Reforma, basado en la idea del destino; otro para la botánica, físico y terapéutico, y otro más para la mitología y sus metamorfosis (51-52). García Mahiues llega a la conclusión de que los múltiples significados crecieron en forma arbórea "en función de condiciones diferentes" (841).

La emblemática floral de los italianos Pierio Valeriano y César Ripa permeó a los emblemistas españoles vinculándose al programa ascético contrarreformista. Entre los que conservaron mucho de la frescura humanista previa al Concilio de Trento, habría que señalar, por su marcado influjo en artistas y escritores del Siglo de Oro, a Sebastián de Covarrubias con sus Emblemas morales. Pardo Lesta menciona a Solórzano Pereira, Núñez

\footnotetext{
${ }^{1}$ Abentofáil escribió su fábula en la España árabe del siglo XII.

${ }^{2}$ La sainteté de vie tirée de la considération des fleurs. Lieja, 1641 y 1653. (29, nota 51).
} 
de Cepeda y Saavedra Fajardo como emblemistas que retoman el motivo del jardín y su simbolismo, pero es Covarrubias quien incluye en sus Centurias tres flores concretas, las cuales aparecerán en los sonetos de Luis de Sandoval Zapata: la rosa, la azucena y el girasol (104 y 112). Entre ellas faltaría el clavel, que tampoco aparece en el admirable repertorio emblemático que Rafael García Mahiues ha recopilado en su tesis doctoral Flora emblemática, aproximación descriptiva del código icónico, ${ }^{3}$ del cual me valdré más adelante gracias a la generosidad del investigador, quien me facilitó su trabajo. El clavel, no obstante, es mencionado por Julián Gállego (192193) como emblema del Amor Humano, ${ }^{4}$ lo es también de las alianzas y del matrimonio, mientras que para el cristianismo será el Amor Divino representado por la Virgen.

Con el antecedente de la "Descripción de la Abadía o Jardín del Duque de Alba"(Vega Carpio: 84-93) ${ }^{5}$ de Lope de Vega, aparecida en la segunda parte de las Rimas, en 1604, así como el "Huerto deshecho" del mismo autor, los poetas españoles del siglo XvII compartieron la idea salesiana del paseo por el jardín como motivo de inspiración sobre la perfección de la obra divina (Tapié: 26). Falkengburg, por su parte, explora un tratado flamenco de $1548^{6}$ en el que el alma vaga deleitada en un jardín de flores y frutos, donde el acto de cortarlos, olerlos y probarlos, le permite participar de la obra divina (36). En esos paseos poéticos eran frecuentes las menciones desordenadas y hedonistas de diversas flores. Así sucede en la Arcadia, de Lope. Woods comenta cómo la poesía barroca se vale de largas enumeraciones de objetos como modelo de descripción topográfica (87-89). Este método aproximativo es muy evidente también en el largo romance "A la Creación Divina", donde Lope coordina asindéticamente extensos repertorios vegetales. De hecho, la acumulación de elementos es un rasgo manierista; en ese mismo año de 1604 apareció La grandeza mexicana, de Bernardo de Balbuena, donde podemos apreciar una total coincidencia estilística con este procedimiento

\footnotetext{
${ }^{3}$ Véase nota 14 y Bibliografía.

${ }^{4}$ En la introducción de Bernard Mercier a Fleurs et Jardins dans l'Art Flamand, Gand, 1960, citado por Gállego: 192, nota 38.

${ }^{5}$ Pardo Lesta ha nombrado las obras de Lope, Jacinto Polo, Góngora, Argensola y otros en relación con el jardín y su simbolismo (1027-1028 y 1033-1034).

${ }^{6}$ Een seer schoen devoet boecxken gheheten der Minnergaert [Un bello y piadoso libro titulado El Jardin del Amor] (33).
} 
de Lope, sobre todo en el Capítulo VI, "Primavera mortal y sus indicios" (94-99). La acumulación nominal de vegetales cede luego paso, entre los poetas, a una semantización mayor de los elementos mencionados. Ya en el romance "Del Palacio de la Primavera", de Luis de Góngora (6465), podemos ver cómo los atributos de las flores las van dotando de una densidad semántica próxima al emblema:

\author{
Esperando están la rosa \\ Cuantas contiene un vergel \\ Flores, hijas de la aurora, \\ Bellas cuanto pueden ser... \\ Como a reina de las flores, \\ Guarda la cińe fiel, \\ Si son arcas las espinas \\ Que en torno de ella se ven \\ $[\ldots]$ \\ Los colores de la reina \\ Vistió galán el clavel, \\ Príncipe que es de la sangre, \\ $\mathrm{Y}$ aun aspirante a ser rey... \\ Las azucenas la sirven \\ De dueñas de honor, y a fe \\ Que sus diez varas de holanda \\ Las invidian más de diez.
}

Pero aquí Góngora, en lugar de la emblematización cristiana, convierte a las flores en personajes de una corte paganizada, donde la rosa es reina; el clavel, galán, y la azucena, dama de honor. Sólo al final asoma la clásica alusión ejemplar a la breve existencia del jardín. Por su parte, Francisco de Rioja, el poeta llamado "de las flores", aprovechó más los significados emblemáticos de ellas en sus Silvas, entre las cuales las dedicadas "A la rosa", "Al clavel", "A la rosa amarilla", "Al jazmín" y "A la arrebolera" (381-383) privilegian, como en el caso de Góngora, las alusiones mitológicas. Sólo el dedicado a la rosa y a la arrebolera se refieren a la vanitas: 


\begin{abstract}
A LA ROSA
Bañóte en su color sangre divina

De la deidad que dieron las espumas;

Y esto, purpúrea flor, y esto ¿no pudo

Hacer menos violento el rayo agudo?...

Que dudo si en sus lágrimas la aurora

Mustia tu nacimiento ó muerte llora.
\end{abstract}

En los demás el poeta encuentra ocasión de explayar sus más íntimos sentimientos, señaladamente en el caso del clavel. ${ }^{7}$ Mientras tanto, en la arrebolera o sandiego de noche, Rioja explora la metáfora de la nave que la flor navega durante el día, que Sandoval Zapata atribuirá al girasol en su soneto 28, "Girasol que, al pimpollo desunido..." (107), llamándolo "timón" y aludiendo a su navegación tras la luz solar. Dice Rioja:
A LA ARREBOLERA
...No inquietes atrevida
El cano seno á los profundos mares,
Que por ventura negarán camino
En daño tuyo á tu serrado pino,
$Y$ en vez de la acogida
Que en las pardas entrañas
Hallaste siempre de la tierra dura,
Hallarás en sus aguas sepultura.

\footnotetext{
7 Particularmente en los últimos versos:

Si al dulce labio llegas, que provoca

A suave deleite al más helado,

Luego que tu encendido seno toca,

A tu color sangriento

Vuelves jay, oh dolor! mas abrasado.

¿Dióte naturaleza sentimiento?

¡Oh yo dichoso á habérseme negado!

Hable mas de tu olor y de tu fuego

Aquel á quien envidias de favores

No alteran el sosiego.
} 
Es precisamente Rioja con quien más afinidades parece tener Sandoval Zapata, pese al predominio absoluto del tópico de la vanitas en los sonetos de este último sobre las flores. Jacinto Polo es otro precedente de Sandoval. Sus Academias del jardin (1931: 105-139), en prosa y en verso, de 1630 , contienen en varios metros pequeños poemas a la azucena, al mirto, a la rosa, a la maravilla, a los claveles, a las clavellinas de Indias o terciopelo, al narciso y al girasol o "flor de sol". En ellos se despliegan los significados emblemáticos frecuentes: la castidad de la azucena:
nieve del mayo, madrugaste cana, con alma de oro castidad vestida, sin que tache una espina tu pureza, rondada del arroyo tu belleza y tu alma del hombre pretendida.

La muerte infausta y ejemplar del mirto:

$$
\begin{aligned}
& \text { porque infausta historia } \\
& \text { verde conserve el prado en la memoria; } \\
& \text { y trueca en mirto Polidoro, el nombre, } \\
& \text { para que enseńe tu desdicha al hombre. }
\end{aligned}
$$

Los claveles como galanes o príncipes:

los que al príncipe del día toga de púrpura ofrecen<smiles>[CH]1[CH]C[CH]1</smiles>

galanes de esotras flores, los lindos de los vergeles [...] que en los solares del prado noble ejecutoria tienen.

Y la piedad del narciso:

Narciso bello, que en papel bruñido, o en lienzo transparente, del cristal detenido de una fuente 


\begin{abstract}
copias tu original, que te enamora, sordo al peńasco, que con voz te llora, y al monte, que con ecos te suspira; si el que no te merece te retira (pues ninguna nació para igualarte, y nadie espera tan hermosa suerte) no lleguen por tu mérito a alcanzarte, lleguen por tu piedad a merecerte.
\end{abstract}

Todos estos sentidos serán comentados en relación con los sonetos de Sandoval. Por ahora sólo conviene señalar que en Jacinto Polo está notoriamente ausente el tema de la vanitas, y el poeta se regodea con holgura en la belleza sensual de las flores, salvo el caso aislado del mirto, que la tradición emblemática asocia a la Pasión de Cristo. En sus Ocios de la Soledad, de 1633 (1948:165-175), describe Jacinto Polo la serenidad de un paseo por un jardín donde las flores son de nuevo mencionadas todas juntas en el largo espacio de la silva. Su emblematismo recuerda también a Góngora por su sensualidad pagana sin pesadumbre neoestoica. Por su parte, la descripción en tercetos endecasílabos de los jardines de Aranjuez, de Lupercio Leonardo de Argensola, aparecidos en sus Rimas (153-160), de 1634, comparten la ékphrasis del jardín, aunque sin detenerse de manera individual en las flores. Por fin, en 1652, el Paraiso cerrado para muchos, jardin abierto para pocos, de Pedro Soto de Rojas (128), inspirado en las descripciones topiarias de la Hypnerotomachia de Polifilo (Pardo Lesta: 1024) reproduce la escala en espiral al cielo por siete mansiones o "terrazas", a través de las cuales transita el alma del poeta. Ya he mencionado antes esta obra de Soto de Rojas como un probable antecedente del Primero sueño, de sor Juana, sobre todo por su carácter de silva emblemática (Olivares 2005: 1283-1294). ${ }^{8}$ Ahí las flores comparten los significados alegres de Góngora y Jacinto Polo: la rosa es reina y el clavel galán, pero la azucena no puede desprenderse de la significación de pureza y virtud cristianas, mientras que el girasol se apega a su vez a la connotación mitológica:

${ }^{8}$ Con anterioridad, sólo Emilio Orozco Díaz (42) llega a asociar a Soto y Rojas y a Sor Juana como cultivadores de la silva descriptiva. 
Purpúreo dio su parecer la rosa, con estoques cubiertos defendido [...]

Más galán el clavel que presumido, de grana se advirtió, y el ámbar vestido; y por su prosapia, lado a lado, salió la melotisa de encarnado; niña traveseando, la violeta se levantó con el olfato ufana; jacinto en esta corte adelantado de Telemón, que es último trofeo, la sigue, y su color, con galanteo [...]

El alhelí, cuya virtud estima el fármaco atendido, si trasciende su fama, en la vista se asienta y alentado gabán de gualda ostenta [...]

Sucesión de alta rama, el nardo que, eminente primer rey escogido, ser pudiera cetro galán del pueblo de las flores, plateados olores esparce en la que alcanza media esfera; blando salió el narciso que un instante a su vida forma un lustro [...]

De noche escandaloso, aunque de día bizarreando, el azahar salía, mientras la madreselva se enlazaba, süave se prendía, y el sitio con pastillas perfumaba [...] En su eclíptica, Clicie el movimiento observa de su amor acelerado [...]

Grande en España el tulipán sediento, entre inmensas riquezas de colores, la virtud olvidó de los olores [...] 
Todas ellas habitan y adornan la séptima y última mansión, donde el alma al fin reposa en deleitoso clímax contemplativo. Es sobre todo esta última mansión donde los lectores nos vemos presentes en un verdadero jardín emblemático donde cada hierba y cada gota de agua es un vivo reflejo de la armonía divina.

Luis de Sandoval Zapata revela muchas señas de identidad con los vates andaluces del barroco gongorista: Rioja, Góngora, Jacinto Polo y Soto de Rojas son el contexto poético inmediato que nos permite en gran medida explicar la obra del poeta novohispano. Si bien el conceptismo quevediano es una constante a lo largo de sus sonetos, hay una clara voluntad del poeta por hacer eco de los motivos y formas de sus coetáneos del sur de la península. Por otra parte, la obra emblemática de Sebastián de Covarrubias es otro de sus sustratos más evidentes, además de la tradición del emblematismo cristiano propagado por la Compañía de Jesús. De los veintinueve sonetos que se conservan de Sandoval, doce están dedicados a las flores, es decir, casi la mitad. En ellos Sandoval Zapata toma dos caminos representativos: uno es mencionar con toda claridad la flor de la que se trata en cada soneto, lo que sucede en cinco de ellos, dedicados a la rosa, al clavel, al girasol y dos a la azucena. De los otros siete sonetos, en tres la técnica poética que adopta Sandoval es similar a la de los enigmas difundidos por los cancioneros poéticos (Madrigal: 127-133), los cuales brindan al lector una descripción cuyo motivo hay que adivinar ya sea por el poema, ya por la imagen que se describe al principio con un epígrafe como "Píntase [...]" o "Hase de pintar una mulata de gran boca, sin braços, cruzados los pies, con dos brazos, que se los quiere desuiar". A continuación el poema va desglosando prolija y metafóricamente las cualidades y atributos de lo "pintado" con palabras en el epígrafe, ${ }^{9}$ para que al final el lector u oyente adivine lo que el título del enigma dice como respuesta: "De las tenazas". De faltar u ocultarse el título, desde luego, el lector tiene que echar vuelo

${ }^{9}$ El poema enigmático dice así: Ya podran echar de ver / para lo poco que soy, / pues siruo solo de comer. / Muriera de traspillada / si de comer se me diera, / quando yo se lo pidiera, / mas al fin como forçada. / Que es tal el negro manjar, / y tan por fuerça le tomo, / que primero que le como, / los ojos me han de tapar. / De ordinario como assado, / y tal a comerlo vengo, / que gran rato me detengo / entre bocado y bocado. / Y el mal olor recebido / por momentos me prouoca / a que buelua por la boca / esso poco que he comido. / En ayunas suele ser / el olor de boca en vnas, / mas en mi no es en ayunas, / sino despues de comer. 
a la imaginación a partir de las metáforas para dar con la respuesta. En estos casos el parentesco con el emblema es harto evidente, tomando el lugar de la imagen el epígrafe donde se describe con puntualidad la figura; del epigrama, el desarrollo poético del enigma con todas sus alusiones metafóricas, y del mote, el título o respuesta de la adivinanza. Del mismo modo, Sandoval Zapata prescinde de la pintura o su enunciación y omite los títulos, ya sea para referirse a una mera abstracción o para que el lector adivine qué flor está siendo poetizada. Lo último sucede en los sonetos 20,23 y 26 , quedando sólo cuatro sonetos en torno a abstracciones, como una vaga flor carmín, desteñida como sombra platónica, en el soneto 19; otra fugitiva flor blanca en el soneto 22; una flor de múltiples colores posibles, rojo, blanco o rosa, en el soneto 25 , y el "pimpollo" indefinido del soneto 27. En cuanto a los tres que ostentan una calidad enigmática además de emblemática, hay que señalar que el primero, el 20, "Flor a quien el Favonio blando bate [...]" (99), va presentando diversas características que lo asocian al narciso:

Flor a quien el Favonio blando bate con tantas lenguas cuantas plumas bellas, madrugaste a parlar con las estrellas, ave de luz con pico de granate.

Peligros son cuantas centellas cate, volcán que sobre el céfiro descuellas; la misma vanidad de tus centellas es munición que a tu beldad combate.

No ansiosa rompas el umbral del nido, mira que para estar anochecida basta el exordio de querer lucirse.

No te escribas período tan florido, porque en estos papeles de la vida más fácil es borrarse que escribirse.

Flor "de luz", amarilla; con forma de estrella, por lo que sale "a parlar" con los astros; con "pico de granate", esto es, el estigma central en forma de campana; vanidosa de sus "centellas", como el narciso, pero, sobre todo, que se escribe en su "período tan florido", donde, según el poeta, es 
más fácil "borrarse que escribirse". García Mahiques anota que uno de los nombres científicos del narciso es Narcissus poeticus, basado en la nomenclatura de Teofrasto cuando habla del narciso de los poetas (533). Es muy posible que en este soneto el poeta se estuviese refiriendo a sí mismo como tal. Por otro lado, su sentido funerario viene de su identificación con los narcóticos, cuyo nombre genérico se deriva precisamente del narciso e infunden un sueño parecido a la muerte, ciñéndose con coronas de narcisos a los muertos. En este sentido puede hablarse de un narciso "piadoso", como lo hace Jacinto Polo, pues se creía que adoptaba el luto de Démeter cuando se lamentaba por la pérdida de Perséfone (535). ${ }^{10}$ Con más aplicación aún a nuestro soneto, García Mahiques ilustra con un emblema de Alciato (262) $)^{11}$ el significado que el narciso tiene de la philautia, es decir, el amor a las propias doctrinas despreciando la sabiduría antigua. La censura de Sandoval a su flor del soneto 20 se basa precisamente en la audacia de escribirse sin escuchar el sabio consejo sobre su vida pasajera.

En el soneto 23 (102), a lo largo de todas las estrofas se va desarrollando el tema de la muerte:

Con vergüenza se asoman al oriente

flores que abren los párpados tan rojos;

no ven la muerte tan lucidos ojos

porque tienen muy cerca el occidente.

Para presidio del coral viviente

¿qué le ha importado su cancel de abrojos?

para pasarse a fúnebres despojos

son los alientos de la muerte puente.

Si han de apagaros, olorosas llamas, los soplos de la más pálida fuente ¿qué importa con el sol el valimiento?

Más que vosotras viven vuestras ramas, que haber durado más para la muerte es más tardanza, no mayor aliento.

\footnotetext{
${ }^{10}$ Respecto a las significaciones relativas a la mitología griega, García Mahiques refiere a Pausanias, Descripción de Grecia I, 2.

${ }^{11}$ La referencia de García Mahiques es al emblema LXIX.
} 
En el segundo cuarteto el poeta se pregunta qué le importa al aliento de la muerte el cancel de abrojos de esos "párpados" o pequeñas flores rojas. Por el tamaño y color podríamos pensar en el mirto, flor funeraria desde la Antigüedad, según los comentarios de Valeriano (García Mahiques: 592-593). ${ }^{12}$ Pero los abrojos o espinas nos obligan a optar por las flores del granado, cuya identificación con la Pasión de Cristo era también muy popular, considerando que el árbol del granado ha sido hermanado con la cruz por tradición. Podríamos pensar también en la Passiflora o granadilla, una flor roja dentro de cuya corola la tradición ha querido ver la corona de espinas, los tres clavos y las cinco heridas, todo lo cual le ha valido el nombre de pasionaria. Ni el mismo Francisco Hernández, tan objetivo en sus descripciones botánicas, puede evitar mencionar estos atributos crísticos de la pasionaria (247). ${ }^{13}$ Sólo que esta flor se distingue justamente por la compleja y ostensible fisonomía de su centro, que no es rojo, y además no es pequeña, como lo es la flor del granado, cuya forma y tamańo sí puede evocar a los párpados. El triunfo final de la flor del granado sobre la muerte, pues simboliza la resurrección, contrarresta el énfasis del soneto en el poco respeto que el aliento de la muerte tiene a su "cancel de abrojos", que a su vez representa la corona de espinas.

El soneto 26 (Sandoval: 105) también parece ser un enigma de la azucena:

¿Ves esa flor, ves esa pompa breve, esa del mayo rueda numerosa, en cielo de verdor luz olorosa? Pues tantos riesgos cuantas puntas mueve.

Vegetal blanco, pájaro de nieve, por la región del aire luminosa, corriendo al monumento presurosa, sus exhalados ámbares se bebe.

\footnotetext{
${ }^{12}$ Refiere a Valeriano (I, L, 676-677 y I, LII, 693).

${ }^{13}$ Parece haber una confusión en Hernández entre la granadilla, la cual describe como flor, y la granada, ilustrada y descrita por él como fruta. El botanista toma a ambas como la misma planta.
} 
Con el espejo líquido del yelo,

a lienzo blanco o cristalina alfombra,

en especies y sombras retratada;

Uno parece todo en aquel velo,

lo mismo es la hermosura que la sombra,

lo mismo es el aliento que la nada.

Evoca a la azucena porque es "del mayo rueda numerosa", además de "olorosa”; "vegetal blanco" de "exhalados ámbares", más aún porque en el primer terceto Sandoval la duplica "en el espejo líquido del yelo", tal como Jacinto Polo hace reflejar su azucena en el cristal del agua en Ocios de la soledad:

El armińo verás de una azucena,

En la margen hermosa,

Y otra dentro el cristal, floresta amena,

Fragante espuma es, cisne de Flora (168).

Vale recordar que el mes de mayo peninsular es distinto del americano. Los mayos de Sandoval eran harto cálidos, sobre todo si los pasaba en su ingenio azucarero de Tlaltizapán, entre Cuernavaca y Taxco, sin asomo alguno de aguas que se deshielan. Sin embargo en este soneto comparte el contexto de los delicados versos de Jacinto Polo, añadiendo de suyo que la hermosura y aliento de su azucena son todos uno con su reflejo: esto es, sombra y nada.

De los sonetos en que Sandoval anuncia claramente el nombre de la flor, habría que observar que el soneto 18 , sobre la rosa, concluye en el último terceto que más vale cortarla en su plenitud que dejarla marchitarse:

¡Qué tasada respira una ventura!

Aun sin llegar a dos auroras frías

Topó el hierro fatal tan bella suerte.

Pierde respiraciones y hermosura, Que ha de envejecerse con los días, Mayor mal es la vida que la muerte (97). 
Con lo que el poeta participa de su belleza arrebatándola al rosal, como los místicos paseantes que menciona Falkenburg. García Mahiques anota la creencia de que, cortada, la rosa aumenta su fragancia, al igual que la virtud del santo cuando está difunto. De igual manera, la rosa roja cortada era, según Picinelli, símbolo del sacrificio en la flor de la edad (708-710). ${ }^{14}$

El clavel del soneto 24 de Sandoval, que por fin llega a ser rey de las flores, es incapaz de reinar porque le falta el tiempo:

El sol apenas coronel la dora, cuando el coral se marchitó en su oriente, y abatiendo su púrpura la frente, rey no soy, dice, que he de ser de una hora (103).

El girasol del soneto 28, como dijimos, se convierte en el timón que se aventura "en mar del sol navegar día":

Girasol que, al pimpollo desunido, rompiste cárcel de esmeralda fría por volver a vivir argentería, águila hojosa sobre verde nido.

Pájaro en alas de coral florido, a mucho riesgo tu ambición se fía, ¿qué importa en mar del sol navegar día, si has de desembarcar en el olvido?

Por esa misma luz, por esos rayos, tus mismos pasos tu vivir extinguen; expirarás aunque tu luz alegres.

Parasismos y alientos son desmayos, todos matan y sólo se distinguen que unos son tristes y otros son alegres. (107).

También hay que notar que la metáfora "águila hojosa" aplicada al girasol es precedida por Jacinto Polo en sus Academias del jardin, donde el girasol es "águila de las flores" (116).

${ }^{14}$ Refiere a Picinelli, Mondo Simbolico XI, c. 18, nota 161. 
Por fin, la azucena del último soneto, el 29, sufre un proceso alquímico por la acción solar que la convierte de plata en oro:
Blanca azucena que alumbraste el prado
desplegado tu espíritu flamante, fuiste al alba verdor, al sol diamante, con la voz del aire ruiseńor nevado.
Oro marchito, al cristal ajado, polvo de nieve fue la luz brillante; para buscar el movimiento errante está lo bello de tu ser alado.
¡Oh en poca plata cándido diluvio! Un enemigo a tu beldad esquivo hallaste en el pimpollo que rompiste,
Y con la luz de ese veneno rubio y con el oro, aun cuando estaba vivo, la deuda del morir no redimiste (116).

En estos dos últimos poemas, Sandoval resuelve con notable originalidad conceptual la consideración de las flores, añadiendo un toque o una reflexión propia a la tradición emblemática.

Como breve recapitulación, habría que destacar, en primer lugar, la presencia del emblematismo en la poesía barroca novohispana y las diversas formas de recurso que hacían los poetas de esa fuente paradigmática de símbolos y cifras. En segundo, la identificación o parentesco de Luis de Sandoval Zapata con sus coetáneos del sur peninsular, en cuyo grupo habría que incluirlo como poeta americano. Finalmente, la importancia en la obra de Sandoval del mundo de la naturaleza, que además de ser tópico desengañado, es lectura del mundo creado. La Nueva España ofrecía a Sandoval muchos jardines. De los prehispánicos subsistieron durante el virreinato los de Oaxtepec, Alpuyeca, Chapultepec y Tezcuzingo. Es muy probable que nuestro poeta tuviese oportunidad de vagar como los místicos entre las flores de Oaxtepec o de Alpuyeca mientras su ingenio azucarero se consumía y se avivaban las llamas de su ingenio poético. 


\section{Bibliografía}

Alciato. Emblemas. Manuel Montero Vallejo (ed.). Madrid: Editora Nacional, 1975 [Edición facsimilar de la de Bernardino Daza. Lyon, 1540].

Argensola, Lupercio Leonardo de. Rimas. Edición, introducción y notas de José Manuel Blecua. Madrid: Espasa-Calpe, 1972 (Clásicos Castellanos, 173).

Balbuena, Bernardo de. La grandeza mexicana y Compendio apologético en alabanza de la poesía. Estudio preliminar de Luis Adolfo Domínguez. México: Porrúa, 1985 (Sepan cuantos, 200).

Covarrubias Horozco, Sebastián de. Emblemas morales. Edición e introducción de Carmen Bravo Villasante. Madrid: Fundación Universitaria Española, 1978 [Ediciones facsimilares Centuria I, emblema 5; Centuria II, emblemas 104 y 112].

Falkenburg, Reindart L. The Fruit of Devotion. Mysticism and the imagery of love in Flemish paintins of the Virgin and Child 1450-1550. Amsterdam I Philadelphia: John Benjamins, 1994.

Gállego, Julián. Vision et symboles dans la peinture espagnole du Siècle d'Or. Paris: Éditions Kincksieck, 1968.

García Mahiques, Rafael. Flora emblemática, aproximación descriptiva del código icónico. Valencia: Universitat de Valéncia, 1991 [Servicio de Publicaciones, Tesis Doctorales, microfichas].

Góngora, Luis de. Poesías. Prólogo de Anita Arroyo. 4ª Edición. México: Porrúa, 1986. (Sepan cuantos, 262).

GonzÁlez Zárate, Jesús María. "La visión emblemática del triunfo del alma en la obra de Rubens y Jan Brueghel 'Guirnalda con la Virgen y el niño"”, en Goya. Revista de Arte. 209 (mar.-abr., 1989). 282-290.

Goody, Jack. The culture of flowers. Cambridge: Cambridge University Press, 1933.

Hernández, Francisco. Historia natural de Nueva España, 2 vols. Trad. de José Rojo Navarro. México: Universidad Nacional Autónoma de México, Instituto de Biología, 1959.

Madrigal, Miguel de (comp.). Segunda parte del romancero general y flor de diversa poesía. Valladolid: Luis Sancha, 1605.

Morales Folguera, José Miguel. "El viaje neoplatónico y su imagen en los jardines de El Retiro de Málaga”, en Del libro de emblemas a la ciudad simbólica. Actas del III Simposio Internacional de Emblemática Hispánica, vol. I. Víctor Mínguez (ed.). Castelló de la Plana: Universitat de Jaume I, 2000. 323-324. 
Olivares Zorrilla, Rocío. “El libro metágrafo de Alejo de Venegas y El sueño de sor Juana: la lectura del universo", en Anales del Instituto de Investigaciones Estéticas, 76. México: Universidad Nacional Autónoma de México, 2000. 89-112.

—. "Precursores en la sombra: los otros modelos del Primero Sueño, de Sor Juana Inés de la Cruz”, en las Actas del Congreso El Siglo de Oro en el nuevo milenio, II. Carlos Mata y Miguel Zugasti (eds.). Pamplona: Eunsa, 2005. 1283-1294.

Orozco y DíAz, Emilio. Introducción a un poema barroco granadino. De las "Soledades" de Góngora al "Paraíso" de Soto y Rojas. Granada: Universidad de Granada, 1955.

Pardo Lesta, Rubén. "Del mundo simbólico al mundo poético: el Paraíso cerrado de Pedro Soto de Rojas como ejemplo del poema emblemático", en Del libro de emblemas a la ciudad simbólica. Actas del III Simposio internacional de Emblemática hispánica, vol. I. Víctor Mínguez (ed.). Castelló de la Plana: Universitat de Jaume I, 2000. 1009-1034.

Polo de Medina, Salvador Jacinto. Obras escogidas. Estudio, edición y notas de José María de Cossío. Madrid: Compañía General de Artes Gráficas, 1931.

—. Obras completas. Murcia: Tip. Sucesores de Nogués, 1948 (Biblioteca de Autores Murcianos, I).

Rioja, Francisco De. Poetas líricos de los siglos XVI y XVII, I. Edición de Adolfo de Castro. Madrid: Imprenta de Parlado, Páez y Compañía, 1903 (Biblioteca de Autores Españoles, 32).

Sandoval Zapata, Luis De. Obras. Estudio y edición de José Pascual Buxó. México: Fondo de Cultura Económica, 1986 (Letras Mexicanas).

Soto de Rojas, Pedro. Paraiso cerrado para muchos, jardines abiertos para pocos. Los fragmentos de Adonis. Edición de Aurora Egido. Madrid: Cátedra, 1981 (Letras Hispánicas, 128).

Tapié, Alain. Le sens caché des fleurs. Symbolique \& botanique dans la peinture du XVIIIe. siècle. Paris: Adam Biro, 1997.

Valeriano Bolzani y Giovanni Pierio. Hieroglyphica, sive. De sacris aegyptiorum literis commentarii. Basilea, 1556.

Vega Carpio, Lope FÉlix. Obras selectas, II. Estudio preliminar, biografía, bibliografía, notas y aportaciones de Federico Carlos Sáinz de Robles. México: Aguilar, 1994 (Grandes Clásicos).

Woods, M. J. The Poet and the Natural World in the Age of Góngora. Londres: Oxford University Press, 1978. 